\title{
Pneumococcal meningitis: A 12 year experience in a children's hospital prior to the universal immunization with a conjugate vaccine
}

\author{
Griselda Berberian, M.D., ${ }^{a}$ M. Guadalupe Pérez, M.D., ${ }^{a}$ Carolina Epelbaum, M.D., ${ }^{a}$ \\ María del Carmen Ceinos, Biochemist, ${ }^{b}$ Horacio Lopardo, M.D., ${ }^{b}$ and \\ María Teresa Rosanova, M.D. ${ }^{a}$
}

\begin{abstract}
Introduction. Pneumococcal meningitis is caused by Streptococcuspneumoniae and hashigh morbidity and mortality rates. The objective of this study was to identify the epidemiological and clinical characteristics, antibiotic sensitivity and evolution of pneumococcal meningitis in children prior to the introduction of the vaccine in Argentina.

Methods. Patients younger than 18 years old hospitalized atHospitalJ.P.Garrahan between 1999 and 2010 were included. Children's microbiology lab records and case records were reviewed.

Results. One hundred and eleven children with S. pneumoniae meningitis were identified. Forty cases were found in the 1999-2002 period, 35 in the 2003-2006 period, and 36 in the 20072010 period. The mean age was 7 months old (range: 1-191). One hundred and four patients were immunocompetent $(94 \%)$. Only 20 patients $(18 \%)$ had an underlying disease. The most commonly observed clinical presentation was neurological involvement in 80 patients $(75 \%)$, and sepsis in $59(53 \%)$. Forty-nine patients ( $44 \%$ ) had to be admitted to the ICU. A second clinical source of infection was identified in 24 patients (22\%); half of these cases corresponded to pneumonia. Positive findings were observed in the cerebrospinal fluid culture of 103 patients $(93 \%)$ and in the blood culture of $88(79 \%)$.

Resistance to penicillin was identified in $15 \%$ of cases, while $5 \%$ showed resistance to cefotaxime. Antibiotic resistance was reduced over the years. Complications occurred in 56 patients $(50 \%)$, and $11(10 \%)$ died because of the infection.
\end{abstract}

a. Division of Epidemiological Control and Infectology.

b. Division of Microbiology. Hospital de Pediatría "Dr. Prof. Juan P. Garrahan." Buenos Aires, Argentina.

E-mail Address: M. G. Pérez, M.D.: guaperez@hotmail.com

Conflict of interest: None.

Received: 1-13-2014 Accepted: 3-14-2014 countries, with a large number of survivors with sequelae. ${ }^{2}$

The primary etiologic agents of bacterial meningitis in children include Streptococcus pneumoniae, Neisseria meningitidis and Haemophilus influenzae type b. ${ }^{3}$ Its frequency varies depending on theepidemiological and demographic characteristics, and the immunization schedule implemented in each region.

In Argentina, based on data published by the National Ministry of Health of Argentina in 2012, S. pneumoniae was still the main causative etiologic agent of bacterial meningitis. ${ }^{4}$ Recent population studies have assessed the impact of pneumococcal meningitis in Argentina and found that $S$. pneumoniae was the cause of meningitis in $27 \%$ of patients with hearing loss, in $15 \%$ of patients with profound deafness, and in 17\% of patients with mental retardation. ${ }^{5}$

The sensitivity of S. pneumoniae to antimicrobials has changed over the past years. Up-to-date knowledge regarding antibiotic sensitivity in the population is necessary in order to be able to indicate an adequate empiric antibiotic therapy when pneumococcal meningitis is suspected.

The objective of this study was to evaluate theepidemiological, clinical and evolutionary characteristics of children with pneumococcal meningitis and to establish the evolution of antimicrobial sensitivity prior to the introduction of the conjugate vaccine in the Argentine immunization schedule in 2011.

\section{MATERIAL AND METHODS}

This was a retrospective, observational study. All patients younger than 18 years old admitted to the hospital 
with a diagnosis of $S$. pneumoniae meningitis between January $1^{\text {st }}, 1999$ and December $31^{\text {st }}, 2010$ were included. Inclusion criteria were that children had to be younger than 18 years old, had to be hospitalized with the diagnosis of acute meningitis or meningoencephalitis, and S. pneumoniae had to be identified in cerebrospinal fluid (CSF) and/or blood culture samples. Patients diagnosed with secondary meningitis, ventriculo peritoneal shunt or postoperative meningitis and those who were pneumococcus positive in the latex agglutination test with no microbiological isolation were excluded.

Data were obtained by reviewing clinical records and microbiological tests. Demographic, clinical and evolutionary characteristics were recorded, together with antimicrobial sensitivity in each case. Thirty day post-discharge mortality and neurological sequelae at the time of discharge were assessed. The study was conducted in accordance with health research ethical principles.

Microbiological aspects: S. pneumoniae was characterized because it displayed the typical morphology by Gram staining and because it presented alpha hemolysis in Columbia Agar plates with $5 \%$ sheep blood. Final identification was conducted by growth inhibition in optochin discs (Britania Lab, Argentina), solubility in bile salts and agglutination with latex particles (Directigen, Becton Dickinson, Cockeysville, Maryland, USA). All strains showed penicillin sensitivity in 1- $\mu$ g oxacillin discs, according to the recommendations of the Clinical and Laboratory Standards Institute (CLSI), USA. The minimum inhibitory concentration (MIC) to penicillin and third- generation cephalosporins was determined by the Etest method (Biomérieux, Argentina).

Sensitivity to penicillin and third-generation cephalosporins was determined as per the cut- off points suggested by the CLSI for meningitis: ${ }^{6}$ MIC for sensitivity $\leq 0.06 \mu \mathrm{g} / \mathrm{mL}$ and MIC for resistance $\geq 0.12 \mu \mathrm{g} / \mathrm{mL}$. For third-generation cephalosporins, Pneumococci were considered sensitive if the MIC was $\leq 0.5 \mu \mathrm{g} / \mathrm{mL}$ and resistant if the MIC was $\geq 2 \mu \mathrm{g} / \mathrm{mL}$. Pneumococci showing $\mathrm{MIC}=1 \mu \mathrm{g} / \mathrm{mL}$ were considered as having intermediate sensitivity.

\section{RESULTS}

One hundred and eleven children with a clinical presentation compatible with $S$. pneumoniae meningitis and who tested positive for this condition in CSF and/or blood cultures between January $1^{\text {st }}, 1999$ and December $31^{\text {st }}, 2010$ were included. Their mean age was 7 months old (range: $1-191$ months); 58 patients (54\%) were girls. One hundred and four patients were immune competent $(94 \%)$. Only 7 patients $(6 \%)$ had some sort of immune compromise: nephrotic syndrome in 4 patients, HIV in 1 patient, and AML in 1 patient.

Twenty-three patients (18\%) had an underlying disease, and the most common were congenital heart disease in 7 patients $(6 \%)$, central nervous system conditions in 3 patients (3\%), and congenital anomalies with no heart disease in 3 patients (3\%).

Only 2 patients ( $2 \%$ ) had received a pneumococcal vaccine. The mean evolution prior to diagnosis was 2 days (range: 1-7).

Forty-nine patients $(44 \%)$ had to be admitted to the ICU, with a mean length of stay of 5 days; 33 patients $(30 \%)$ required mechanical ventilation.

At the time of admission to the hospital, all children had fever, and 80 patients $(72 \%)$ had at least one neurological symptom. In this series, an altered state of consciousness was the most commonly observed sign. Meningitis was associated with a second clinical source of infection in 24 patients (22\%): pneumonia in 12 patients $(50 \%)$, otitis media in 7 patients $(29 \%)$, periorbital cellulitis in 2 patients $(8 \%)$, sinusitis in 2 patients $(8 \%)$, diarrhea in 2 patients $(8 \%)$. Sepsis developed in 59 patients $(53 \%)$. The mean duration of fever was 3.5 days (range: 2-15).

Lab tests upon admission showed a mean white blood cell count of $15900 / \mathrm{mm}^{3}$ (range: 5-45 000) and a erythrocyte sedimentation rate in the first hour of $80 \mathrm{~mm}$ (range: 40-120).

CSF cytochemistry showed the following mean values: 1335 cells $/ \mathrm{mm}^{3}$ (range: 0-10 000), glucose $28 \mathrm{mg} / \mathrm{dL}$ (range: $0-51$ ) and proteins $250 \mathrm{mg} / \mathrm{dL}$ (range: 11-967). Predominance of polymorphonuclear leukocytes was observed in $82 \%$ ( $n=91$ patients). At the time of admission, a computed tomography scan of the brain was indicated in 84 patients $(77 \%)$, which was pathological in 56 patients (66\%).

The initial empiric treatment was adequate in 107 patients (96\%). In all cases, third-generation cephalosporins were used as first-line treatment. In addition, vancomycin was added to the treatment in 22 patients $(20 \%)$. The mean duration of antibiotic therapy was 13 days (range: 1-45). Corticosteroids were administered before antimicrobials in 100 patients $(90 \%)$.

At least one complication related to meningitis was observed in 58 children (52\%). 
The most common complications were subdural fluid collection in 24 patients $(46 \%)$, noncommunicating hydrocephalus in 7 patients $(13 \%)$, vasculitis in 3 patients $(6 \%)$, subdural empyema in 1 patient (2\%), and brain abscess in 1 patient $(2 \%)$.

The mean length of stay in the hospital was 18 days (range: 1-150). Thirty day post-event mortality was $10 \%$ (11 patients).

Thirty-six patients (33\%) had permanent neurological sequelae: developmental delay in 10 patients $(9 \%)$, epilepsy in 6 patients $(5 \%)$, hemiparesis in 6 patients $(5 \%)$, and cranial nerve involvement in 4 patients (4\%). During follow-up, hearing was assessed only in 54 patients, and $10(19 \%)$ showed certain degree of hearing involvement.

S. pneumoniae was isolated in all patients: in the CSF of 103 patients (93\%) and in the blood culture of 88 patients $(79 \%)$. Upon identification, $94(85 \%)$ strains were sensitive to penicillin and 105 (95\%) were sensitive to thirdgeneration cephalosporins.

By dividing the study into three subsequent periods of 4 years each (period I: 1999-2002; period II: 2003-2006; period III: 2007-2010), it was observed that penicillin resistance was reducing throughout all periods: $10 / 40(25 \%)$ over period I, 5/35 (14\%) over period II, and 2/36 $(5 \%)$ over period III. However, resistance to thirdgeneration cephalosporins was only observed in the first period (6/40 cases). As seen all over the world, no vancomycin-resistant $S$. pneumoniae was identified.

\section{DISCUSSION}

Invasive pneumococcal disease is one of the main causes of morbidity and mortality in the pediatric age group. At present, in Argentina, $S$. pneumoniae is the main causative agent of bacteremia and bacterial meningitis in children. ${ }^{3}$

Meningitis, together with sepsis, are the most severe clinical forms of invasive $S$. pneumoniae disease given its high mortality and associated sequelae rates. ${ }^{4}$ Prior to the introduction of the pneumococcal conjugate vaccine, invasive S. pneumoniae meningitis was the prevalent etiology in children younger than 2 years old. ${ }^{7}$ Pneumococcal meningitis has been reported in even younger children. ${ }^{8,9}$ In agreement with the literature, in our series the mean age of patients was 7 months old.

Even though immune system disturbances, a history of basilar skull fracture and CSF fistulae are considered predisposing factors to $S$. pneumoniae meningitis, ${ }^{10}$ most cases are observed in previously healthy children. The frequency of comorbidities identified in the literature ranges from $13 \%{ }^{11}$ to $32 \% .^{12}$ In this study, $18 \%$ of children had an underlying disease.

The most common neurological sign of $S$. pneumoniae meningitis described in the literature and in this study was an altered state of consciousness. ${ }^{11}$

An increase in the number of cells observed in CSF, above 500 cells $/ \mathrm{mm},{ }^{3}$ hypoglycorrhachia and hyperproteinorrhachia have been consistently described in the different series of patients with $S$. pneumoniae meningitis. ${ }^{9-13}$ Geiseler, et al. ${ }^{13}$ studied 1064 patients with bacterial meningitis and only $1.5 \%$ had normal cellularity. In this study, all patients who had meningitis without pleocytosis corresponded to Neisseria meningitidis meningitis.

Complications and secondary morbidity are the most serious problems associated with meningitis. Some studies have found a higher rate of neurological complications in patients whose etiologic agent was S. pneumoniae. ${ }^{14,15}$ A study conducted on 81 meningitis patients at Hospital de NiñosPedrodeElizalde, in the City of Buenos Aires, reported that $60 \%$ of patients with S. pneumoniae meningitis had complications, compared to $22 \%$ of patients with other etiologies. ${ }^{15}$ Abate, et al. ${ }^{16}$ found that $42 \%$ of 221 patients with S. pneumoniae meningitis experienced complications. In this study, half of patients had at least one meningitisassociated complication, while a third of them developed neurological sequelae.

According to the bibliography, mortality associated with pneumococcal meningitis ranges between $2.6 \%{ }^{6}$ and $11.4 \% .{ }^{17}$ In our study, the mortality rate was $10 \%$, similar to other pediatric studies.

Antibiotic resistance by S. pneumoniae varies depending on the local epidemiological characteristics, circulating serotypes, administration of pneumococcal vaccines, and local guidelines on the use of antimicrobials. ${ }^{18}$

The evolution of antibiotic resistance by $S$. pneumoniae in Latin America has been described in the reports issued by the Surveillance System for the Bacterial Agents Responsible for Pneumonia and Meningitis (SIREVA), which has been established to monitor antibiotic sensitivity and predominant serotypes at a regional level. In 2006 in Argentina, 23.3\% of strains of S. pneumoniae meningitis were identified as penicillin-resistant, 
and $5.4 \%$ of pneumococci, as resistant to thirdgeneration cephalosporins. ${ }^{19}$ In 2012 , penicillinresistance was observed in $20.8 \%$ of strains, with no strain being resistant to third-generation cephalosporins..$^{20}$ This is consistent with the reduction of antibiotic resistance in terms of MIC over the study observation period.

In those countries where universal immunization with a pneumococcal vaccine has been implemented, a significant reduction in the number of hospitalizations due to meningitis ${ }^{21}$ and increased non vaccine serotypes have been described. ${ }^{22}$

Based on circulating serotypes, studies conducted in Argentina reported a potential vaccine coverage against invasive infections of $73 \%{ }^{23}$ and $2.2 \%{ }^{24}$ with the 10 and 13 valent conjugate vaccines, respectively. The recent inclusion of this vaccine in the national immunization schedule of Argentina points to the need of continuous surveillance to find out the impact of this action on pneumococcal meningitis epidemiology across the country. ${ }^{25}$

The selection of an empiric antibiotic treatment for S. pneumoniae meningitis depends on penicillin and third-generation cephalosporin resistance patterns observed in each region. According to the results reported in this and other regional studies, ${ }^{19}$ third-generation cephalosporins continue being the recommended antibiotic for primary meningitis in the community for immunocompetent hosts.

The retrospective nature of this study, the difficulty to perform a long-term follow-up to assess sequelae, and the fact that $S$. pneumoniae serotypes of cases included in the study were not identified account for the main limitations of this study.

\section{CONCLUSIONS}

The number of pneumococcal meningitis cases was similar along the 12 year study period, but resistance decreased during the period of study.

The latest finding of S. pneumoniae resistant to thirdgeneration cephalosporins corresponded to 2001. It is necessary to continue with epidemiological surveillance of the number, resistance patterns and circulating serotypes of S. pneumoniae as of the inclusion of the pneumococcal vaccine in the national immunization schedule to evaluate possible modifications in this setting.

\section{REFERENCES}

1. Swartz MN. Bacterial meningitis: a view of the past 90 years. N Engl J Med 2004;351(18):1826-8.

2. Goetghebuer T, West TE, Wermenbol V, Cadbury AL, et al. Outcome of meningitis caused by Streptococcus pneumoniae and Haemophilus influenzae type b in children in The Gambia. Trop Med Int Health 2000;5(3):207-13.

3. Rubinsky R. Infecciones por Streptococcus pneumoniae. En: Paganini H. Infectología Pediátrica. Buenos Aires: Editorial Científica Interamericana; 2007. Págs.975-85.

4. Ministerio de Salud de la Nación Argentina. Secretaría de Promoción y Programas Sanitarios. Boletín Integrado de Vigilancia. N. ${ }^{\circ} 154-S E 2$ enerode2013.Pág. 6. [Accessed:May 14, 2014].Available at: http:/ / www.msal.gov.ar/images / stories/boletines/BoletinIntegradoDeVigilanciaVersion_ N154-SE2.pdf.

5. Giglio N, Micone P, Gentile A. The pharmacoeconomics of pneumococcal conjugate vaccines in LatinAmerica. Vaccine 2011;29(Suppl 3):C35-42.

6. Clinical and Laboratory Standards Institute. Performance standards for antimicrobial susceptibility testing: TwentySecond informational supplement. CLSI document M100-S22. Vol. 32(3). Wayne, PA: Clinical and Laboratory Standards Institute; 2012. [Accessed: March 17, 2014]. Available at: http://antimicrobianos.com.ar/ATB/wpcontent/uploads /2012/11/M100S22E.pdf.

7. Haddy RI,PerryK,ChackoCE,HeltonWB, etal.Comparison of incidence of invasive Streptococcus pneumoniae disease among children before and after introduction of conjugated pneumococcal vaccine. Pediatr Infect Dis J 2005;24(4):320-3.

8. EskolaJ, TakalaAK, Kela E,Pekkanen E, etal.Epidemiology of invasive pneumococcal infections in children in Finland. JAMA 1992;268(23):3323-7.

9. Usen S, Adegbola R, Mulholland K, Jaffar S, et al. Epidemiology of invasive pneumococcal disease in the Western Region, The Gambia. Pediatr Infect Dis J 1998;17(1):23-8.

10. Durand ML, Calderwood SB, Weber DJ, Miller SI, et al. Acute bacterial meningitis in adults. A review of 493 episodes. N Engl J Med 1993;328(1):21-8.

11. Bingen E, Levy C, Varon E, de La Rocque F, et al. Pneumococcal meningitis in the era of pneumococcal conjugate vaccine implementation. Eur J Clin Microbiol Infect Dis 2008;27(3):191-9.

12. Soult Rubio JA, Rangel Pineda C, Muñoz Sáez M, Parrilla Parrilla JS, et al. Meningitis neumocócica: características epidemiológicas, clínicas y bacteriológicas. An Esp Pediatr 2001;55(4):315-20.

13. GeiselerPJ,NelsonKE. Bacterialmeningitiswithoutclinical signs of meningeal irritation. SouthMed J1982;75(4):448-50.

14. Arditi M, Mason EO Jr, Bradley JS, Tan TQ, et al. Threeyear multicenter surveillance of pneumococcal meningitis in children: clinical characteristics, and outcome related to penicillin susceptibility and dexamethasone use. Pediatrics 1998;102(5):1087-97.

15. Davenport MC, Del Valle MP, Gallegos P, Kannemann AL, BokserVS. Meningitis bacteriana: factores de riesgo para el desarrollo de complicaciones agudas. Arch Argent Pediatr 2007;105(5):405-10.

16. Abate H, Apra E, Barros F, Cibau C, Rosaenz A. Morbimortalidad de las meningitis bacterianas infantiles, no neonatales. Arch Argent Pediatr 1999;97(5):300-5.

17. Levy C, Varon E, Bingen E, Picard C, et al. Méningites à pneumocoque de l'enfant en France: 832 cas de 2001 à 2007. Arch Pediatr 2008;15(Suppl 3):S111-8.

18. Castañeda E, Agudelo CI, Regueira M, Corso A, et al. Laboratory-based surveillance of Streptococcus pneumoniae invasive disease in children in 10 Latin American 
countries: a SIREVA II project, 2000-2005. Pediatr Infect Dis J 2009;28(9):e265-70.

19. Informe Regional de SIREVA II, 2006: datos por país y por grupos de edad sobre las características de los aislamientos de Streptococcus pneumoniae, Haemophilus influenzae y Neisseria meningitidis en procesos invasores. Washington, DC: OPS, 2008. [Accessed: March 17, 2014]. Available at: http://www1.paho.org/Spanish/AD/THS/EV/labs_ Sireva_II_2006.pdf.

20. Informe Regional de SIREVA II, 2012: datos por país y por grupos de edad sobre las características de los aislamientos de Streptococcus pneumoniae, Haemophilus influenzae y Neisseria meningitidis en procesos invasores. Washington, DC: OPS, 2012. [Accessed: March 17, 2014]. Available at: http://www. paho.org/hq/index.php?option=com_co ntent\&view=categor y\&layout=blog\&id=3609\&Itemid=39 $53 \&$ lang $=\mathrm{es}$.

21. Tsai CJ, Griffin MR, Nuorti JP, Grijalva CG. Changing epidemiology of pneumococcal meningitis after the introduction of pneumococcal conjugate vaccine in the United States. Clin Infect Dis 2008;46(11):1664-72.

22. Hsu HE, Shutt KA, Moore MR, Beall BW, et al. Effect of pneumococcal conjugate vaccine on pneumococcal meningitis. N Engl J Med 2009;360(3):244-56.

23. Reijtman V, Fossati S, Hernández C, Sommerfleck P, et al. Serotype distribution of pneumococci isolated from pediatric patients with acute otitis media and invasive infections, and potential coverage of pneumococcal conjugated vaccines. Rev Argent Microbiol 2013;45(1):27-33.

24. Pérez GM, Parra A, Casimir L, Mastroianni A, et al. Infecciones invasivas por Streptococcus pneumoniae en un hospital pediátrico de tercer nivel antes de la introducción de la vacuna conjugada. Características clínicas y serotipos involucrados. Arch Argent Pediatr 2013;111(3):202-5.

25. Ministerio de Salud de la Nación Argentina. Resolución 502/2011. [Accessed: March 17, 2014]. Available at http:// www.msal.gov.ar/pronacei/index.php/institucional/ marco-legal/375-resolucion-5022011. 